\title{
BMJ Open Effects of an optimised approach to home-based respiratory care in individuals with amyotrophic lateral sclerosis: a study protocol for a randomised controlled trial
}

\author{
Karen Pondofe (D) , ${ }^{1}$ Guilherme A F Fregonezi, ${ }^{1}$ Ozana Brito, ${ }^{1}$ \\ Mario Emilio Dourado Júnior, ${ }^{2}$ Rodrigo Torres-Castro, ${ }^{3}$ Vanessa R Resqueti ${ }^{1}$
}

To cite: Pondofe $\mathrm{K}$, Fregonezi GAF, Brito 0, et al. Effects of an optimised approach to home-based respiratory care in individuals with amyotrophic lateral sclerosis: a study protocol for a randomised controlled trial. BMJ Open 2021;11:e042780. doi:10.1136/ bmjopen-2020-042780

- Prepublication history and supplemental material for this paper is available online. To view these files, please visit the journal online (http://dx.doi. org/10.1136/bmjopen-2020042780).

Received 16 July 2020 Revised 22 March 2021 Accepted 23 March 2021

Check for updates

(C) Author(s) (or their employer(s)) 2021. Re-use permitted under CC BY-NC. No commercial re-use. See rights and permissions. Published by BMJ.

For numbered affiliations see end of article.

Correspondence to

Ms Karen Pondofe;

karenpondofe@yahoo.com.br

\section{ABSTRACT}

Introduction This study aims to investigate the effects of an optimal home-based respiratory care protocol in individuals with amyotrophic lateral sclerosis (ALS). Methods and analysis This is a randomised, blinded controlled trial involving patients diagnosed with ALS, both sexes, age between 18 and 80 years. Patients will be randomly allocated into the conventional respiratory care (CRC) group and the optimised respiratory care homebased (ORC) group. Primary outcomes will be peak cough flow, the number of exacerbations and ALS Functional Rating Scale Revised. Secondary outcomes will include chest wall volumes, maximal respiratory pressures, sniff nasal inspiratory pressure, nasal expiratory pressure and forced vital capacity (FVC), forced expiratory volume in the 1st second $\left(\mathrm{FEV}_{1}\right)$ and $\mathrm{FEV}_{1} / \mathrm{FVC}$. The CRC group will receive educational information about respiratory care at the clinic. The ORC group will receive conventional care and home-based care. The clinical status of all individuals will be monitored weekly through telephone calls. A 6-month intervention is planned, the outcomes will be assessed every 3 months and 3 and 6 months follow-up after final evaluation. The primary and secondary results will be described as average or median for continuous variables and absolute and relative frequencies for qualitative variables. Treatment effects or differences between the outcomes (baseline, 3 months and 6 months) of the study groups will be analysed using an analysis of variance. The level of significance will be set as $p \leq 0.05$.

Ethics and dissemination The research ethics committee approved the study. It is expected to evaluate respiratory function in patients with ALS in the short, medium and long terms with home-based care protocol applied. The disease's rapid progression is a limitation for performing a long-term clinical study.

Trial registration number RBR-3z23ts; Pre-results.

\section{INTRODUCTION}

Amyotrophic lateral sclerosis (ALS) is characterised by signs and symptoms of degeneration of upper and lower motor neurons. This leads to progressive weakness of bulbar,
Strengths and limitations of this study

- Randomised clinical trial with evaluator blinded to interventions.

- Quantitative and qualitative outcomes.

- Respiratory home-based care.

- Short-term, medium-term and long-term follow-ups.

- The rapid evolution of the disease can prevent longterm monitoring of some participants.

limb, thoracic and abdominal muscles, ${ }^{1}$ causing loss of deambulation and functionality, difficulties in deglutition, respiratory muscle dysfunction and death due ventilatory failure. ${ }^{2}$ It is one of the most disabling neuromuscular diseases, with considerably rapid and fatal progression. ${ }^{3}$

Studies of ALS epidemiology vary in relation to incidence and prevalence rates due to the geographic and demographic diversity of the global ALS population with variable rates of incidence of $0.3-3.6$ cases/100000 persons/ year and prevalence 1.0-11.3 cases $/ 100$ 000 persons. ${ }^{4}$ There are only a few clinicalepidemiological studies about ALS in Brazil. ${ }^{5}$ The incidence of 0.4 cases $/ 100000$ persons/ year and prevalence of $0.9-1.5$ cases $/ 100000$ persons ${ }^{6}$ were estimated on data provided by a study performed in São Paulo city. ${ }^{7}$

In ALS, disease onset often occurs in two distinct ways: limb onset ALS or bulbar onset ALS. Limb onset ALS occurs in most patients with asymmetric and painless weakness. The clinical examination usually reveals atrophy, muscle weakness and fasciculations that indicate involvement of the lower motor neuron impairment. ${ }^{89}$ The presence of hyper-reflexia and mild-to-severe hypertonia, indicating degeneration of the upper motor neuron. ${ }^{9}$ On the other hand, bulbar onset ALS occurs 
in about $20 \%$ of patients. The weakness begins in bulbar muscles, dysarthria, dysphagia and tongue fasciculations. When there is limb hyper-reflexia, it suggests that the disease has already spread. Patients with bulbar onset ALS have a worse prognosis than patients with limb onset, with an average survival of 2 years and long-term survival (>10 years) of only $3 \% .{ }^{89}$ About $3 \%-5 \%$ of patients have respiratory onset ALS with signs of orthopnoea or mild dyspnoea, even with no limb or bulbar signs. The prognosis is an average survival of 1.4 years and no long-term survival. ${ }^{9}$ It is associated with a rapid disease progression and a higher decline of the forced vital capacity (FVC) and respiratory muscle strength. ${ }^{10-12}$

The progression of the disease decreases the patient's physical function and interferes with activities of daily living (ADL). These activities can be assessed using the Revised Amyotrophic Lateral Sclerosis Functional Rating Scale (ALSFRS-R). The ALSFRS-R is a validated scale based on a questionnaire that measures the physical function of performing ADL of patients with ALS, with a more improved assessment of respiratory symptoms, more sensitive to change and has a better ability to predict survival than the original ALSFRS. ${ }^{13}$

The underlying mechanisms of disease progression are related to motor and respiratory functions. Muscle weakness progresses variably and asymmetrically in the upper and lower limbs from the early stages of the disease, and it is characterised by a reduced functional performance that negatively affects the ADL. The progressive loss of respiratory muscle strength is one of the main problems that afflict individuals with ALS. ${ }^{14}$

Respiratory muscle weakness impairs the capacity to expand the rib cage and elastic recoil, which changes lung compliance and reduces total lung capacity, vital capacity and functional residual capacity. ${ }^{15}$ The combination of inspiratory muscle weakness and reduced chest wall compliance limits the operating volume required for effective coughing. This operating volume is the volume of air inspired at the end of the inspiratory cough phase, and it is considered the most critical determinant of peak cough flow (PCF), as it affects the length of the expiratory muscle and its contraction efficiency. When glottis dysfunction is present, adequate pressure for generating compressive forces for the expectoration of airway secretions cannot be attained, and the effectiveness of coughing is further reduced. ${ }^{16-18}$

Physiotherapy plays an important role in monitoring signs and symptoms of respiratory impairment, as very low PCF can accelerate the development of respiratory failure and death in ALS. Signs and symptoms of respiratory impairment include cyanosis, tachypnoea, tachycardia, use of auxiliary respiratory muscles, decreased chest movements, dyspnoea on exertion or talking and peripheral blood oxygen saturation $\left(\mathrm{SpO}_{2}\right)$ below $90 \%$, which is a clinical emergency. ${ }^{19}$

Currently, no cure or effective medical treatment is available for ALS; nevertheless, multidisciplinary symptomatic treatment can be provided. ${ }^{3}$ According to a systematic review, ${ }^{19}$ specific respiratory care interventions, such as inspiratory muscle training, recruitment of lung volume by air stacking and manual cough assistance for coughing, may increase the survival of patients with ALS, easing the symptoms of respiratory failure and should be incorporated to the daily routine of these individuals.

Therefore, patients with ALS' management aims to maintain respiratory function, and it involves all aspects of care, including at home. It should be based on a model that integrates interventions with health education for patients and their families to be safe in applying cough assistance techniques and preventing recurrent complications and, therefore, hospitalisations. This study is aimed at investigating the effects of a home-based optimised protocol respiratory care of individuals with ALS.

\section{METHODS AND ANALYSIS}

\section{Study design}

This study is a controlled, randomised and single-blind clinical trial to be conducted at a single research centre. We will perform both intention-to-treat (ITT) and perprotocol analyses. The research will follow the Standard Protocol Items: Recommendations for Interventional Trials. ${ }^{20}$

\section{Patients}

\section{Inclusion criteria}

We will include patients with (a) the diagnosis of definite ALS, probable ALS, probable ALS-laboratory supported, possible ALS or suspected ALS according to $E l$ Escorial revised criteria, ${ }^{21}$ both sexes, (b) age between 18 and 80 years, (c) good cognitive level ( $\geq 17$ score) on the ALS Cognitive Behavioural Screen scale, ${ }^{22}$ (d) FVC $>50 \%$ of predicted, (e) sniff nasal inspiratory pressure (SNIP) $>40 \mathrm{~cm} \mathrm{H}_{2} \mathrm{O}$, (f) a responsible caregiver who will accept the training and follow the programme, $(\mathrm{g})$ a telephone to contact the assistance team, (h) no history of respiratory physiotherapy for at least a month before the survey and (i) informed consent (IC), in writing or verbally, for participation in the study will be obtained from the participant and may be signed by a witness in case the volunteer cannot write.

\section{Exclusion criteria}

Volunteers with (a) cognitive impairment, (b) cardiac, respiratory or musculoskeletal comorbidity, (c) users of invasive mechanical ventilation and (d) patients with tracheostomy will be excluded from the research.

\section{Recruitment procedure}

The patients will be recruited in a Neuromuscular Diseases Outpatient Service at the University Hospital Onofre Lopes. All participants will be evaluated at the PneumoCardioVascular Lab. The study has a date for the first registration in August/2021 and the last registration in August/2022. 


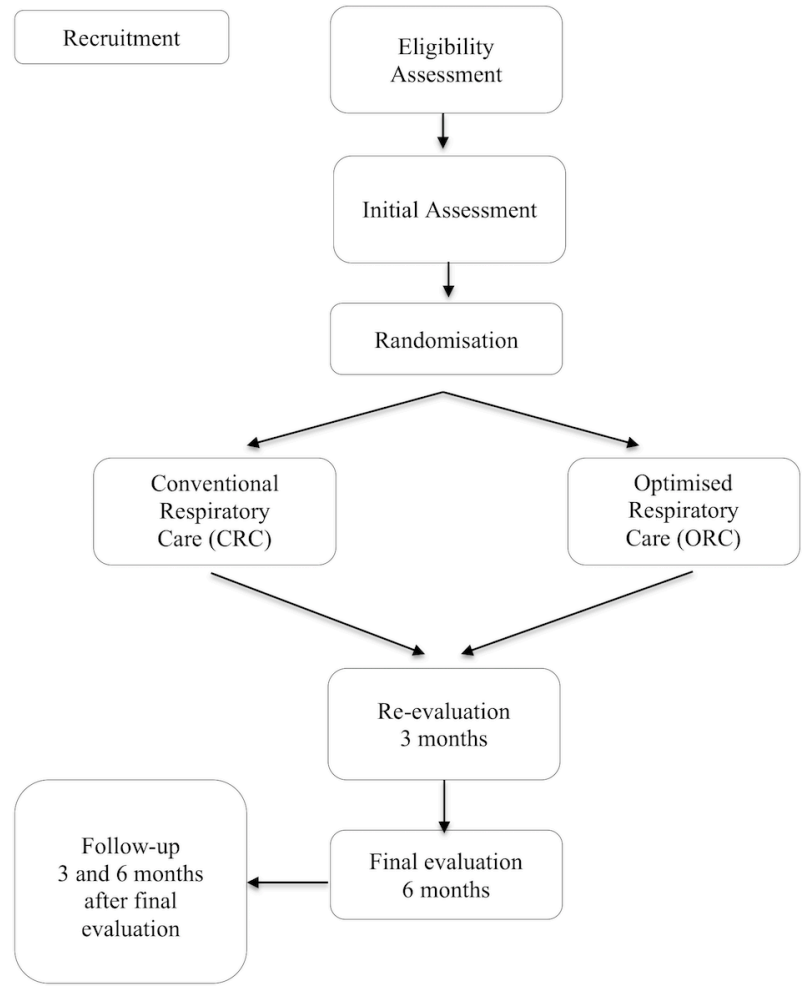

Figure 1 Flowchart of allocation and evaluations of intervention protocols.

Demographic data will include age (years) and gender. Anthropometric data will include height in metres $(\mathrm{m})$ and weight in kilograms $(\mathrm{kg})$. Subsequently, the body mass index, which is the reference measure for obesity control expressed as weight divided by height squared, will be calculated. ${ }^{23}$ Vital signs, including systolic blood pressure, heart rate and respiratory rate, will be monitored manually, and peripheral blood oxygen saturation $\left(\mathrm{SpO}_{2}\right.$, Omron, HEM 7320). ${ }^{24}$

A trained physiotherapist, blinded to the intervention allocation group, will perform the evaluation, which will include lung function, chest wall volumes, PCF, respiratory muscle strength (maximum inspiratory pressure

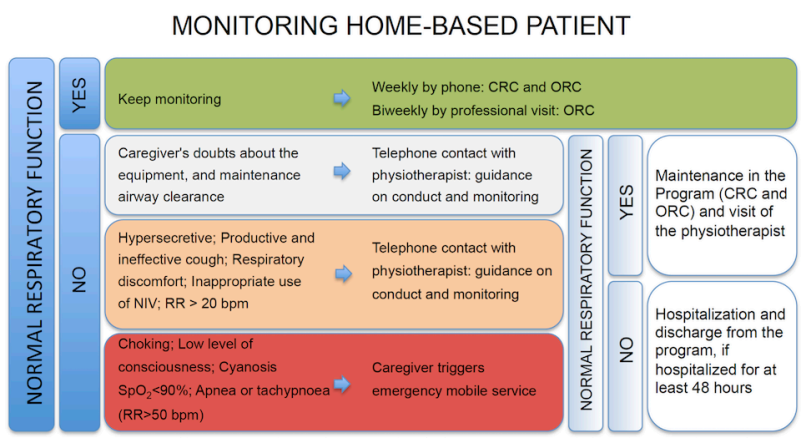

Figure 2 Therapeutic algorithm for respiratory and nonrespiratory events in patients with $\mathrm{ALS}$. $\mathrm{SpO}_{2}, 95 \%-100 \%$; RR, 12bpm-20bpm. ALS, amyotrophic lateral sclerosis; CRC, conventional respiratory care; NIV, non-invasive ventilation; ORC, optimised respiratory care; $\mathrm{RR}$, respiratory rate; $\mathrm{SpO}_{2}$, peripheral oxygen saturation.
(MIP), maximum expiratory pressure (MEP), SNIP and sniff nasal expiratory pressure (SNEP)) and physical function through ALSFRS-R. ${ }^{13}$

Another researcher will use the randomisation system (randomization.com) to allocate participants into two groups after the assessment: conventional respiratory care (CRC) and optimised respiratory care home-based (ORC). The system will initially generate a random numerical list used to allocate the participants in the order of arrival in the study groups before they will receive intervention by a physiotherapist. The same initial evaluator will re-evaluate participants after 3 and 6 months after the beginning of the interventions. A follow-up will be conducted through telephone calls every 3 months until the first year after the beginning of the interventions to assess any exacerbations that may have been caused by emergency care (figure 1).

During the intervention period, all research participants will be followed-up weekly through phone calls to encourage the maintenance of guidelines and provide guidance on the management algorithm designed to determine the best approaches for specific situations (figure 2).

\section{Randomisation procedure and allocation concealment}

The participants will be randomised by an external researcher through a website, 'randomization.com', in four blocks of nine individuals and one block of eight individuals to prevent losses. A list will be generated and used to organise individually sealed and sequentially numbered envelopes. At the beginning of each participant's therapy, the researcher will provide the physiotherapist responsible for the intervention with a coded envelope. This researcher will be the only one with knowledge of each participant's group. After the group allocation, the evaluator will be kept blind during all evaluations and re-evaluations at the PneumoCardioVascular Lab. Participants and caregivers will be instructed not to comment on their treatment. A fourth researcher will be invited to analyse the data at the end of the research.

\section{Intervention groups}

The CRC group will receive education on respiratory care during quarterly hospital visits. The educational information will consist of guidelines on positioning in bed and during fluid and food ingestion as well as the stimulus to mobilisation. The caregiver will be trained on aspirating the oral cavity secretions and assisting patients in coughing through air stacking by manual ventilation with a bag and an oronasal mask (Artificial Manual Breathing Unit-AMBU) when needed. The physiotherapist will provide settings to use or improve non-invasive ventilation and the adaptation of masks, if necessary, on quarterly hospital visits.

The ORC group will receive education on respiratory care during quarterly hospital visits (same that CRC group) and weekly home visits by a physiotherapist. During the visit, the physiotherapist will provide settings 
to use and improve non-invasive ventilation, bronchial hygiene techniques, aspiration of upper airways and assisted coughing through ventilation by mechanical insufflation-exhaustion (CoughAssist E70 Philips Respironics) and/or air stacking.

A trained physiotherapist who is experienced in treating patients with neuromuscular diseases will perform all interventions. Both groups will receive weekly telephone calls to monitor patients and provide the needed assistance to minimise possible complications and exacerbations. Furthermore, all caregivers will be trained to monitor vital signs (systemic blood pressure, heart rate and respiratory rate) and peripheral oxygen saturation.

Weekly monitoring and prior scheduling for consultation with a neurologist during each quarter may help participants to adhere to the study protocols.

\section{Statistics}

\section{Sample size calculation}

Due to the difficulty in conducting clinical trials in people with ALS, the sample size was calculated using data from a previous controlled and randomised clinical trial involving a patient who had a subacute stroke who had glottis dysfunction and difficulty in eliminating secretions. ${ }^{25}$ We calculated, a priori for the investigation of the primary outcome, a total number estimation 44 patients will be divided into two groups. This number was calculated for the primary outcome (peak cough flow $(14.63 \pm 9.48)$ ). Using $G^{*}$ Power software V.3.1.9.2 (University of Düsseldorf, Kiel, Germany), ${ }^{26}$ an analysis of variance analysis was performed, and a dropout rate of $20 \%$ was considered (power $=0.8 ; \mathrm{p}=0.05 ; \mathrm{f}=0.25 ; \mathrm{N}(\min )=36)$.

\section{Statistical analysis}

The Kolmogorov-Smirnov test will be used to analyse the normality of the sample's data. The patient baseline characteristics and outcome variables (both primaries and secondaries) will be summarised using descriptive measures of central tendency and dispersion for quantitative variables, and absolute and relative frequencies for qualitative variables. Possible correlations will be made using Pearson or Spearman tests depending on the normality of the data. Associations will be performed using the $\mathrm{X}^{2}$ or Fischer's exact test. Treatment effects or differences between the outcomes (baseline, 3 months and 6 months) of the study groups will be analysed using an ordinary two-way analysis of variance (ANOVA) will be performed, if the sample is parametric. In case the sample has a non-parametric distribution, paired t-tests and an ordinary one-way ANOVA will be used. Intergroup analyses will be performed using the t-test or the Wilcoxon test, depending on the normality of the data. The analysis will be compared as primary outcomes pre and post treatment of PCF, numbers of exacerbations and ALSFRS-R and secondary outcomes pulmonary function, SNIP, SNEP, MIP and MEP.

The disease progression rate $(\Delta \mathrm{FS})$ will be calculated using a functional scale by the difference between the total value of the ALSFRS-R (48) and the value of the scale in the initial assessment divided by the time (months) since the initial assessment $(\triangle \mathrm{FS}=48$-ALSFRS-R at initial assessment/months). ${ }^{27}$ So, the patients will be classified in slow, intermediated or fast progression if the results of disease progression $\Delta \mathrm{FS} \leq 0.5,0.5 \leq \Delta \mathrm{FS} \leq 1$ and $\Delta \mathrm{FS}$ $\geq 1$, respectively. In case of sufficient data, a subanalysis between groups will be performed.

The analyses will be based on the ITT principle, and they will involve data of all randomised participants with at least one outcome measure. The level of significance will be set as $p \leq 0.05$. All participants will be included in the analysis of the original groups following the Consolidated Standards of Reporting Trials (CONSORT) recommendations. The data will be analysed using GraphPad Prism software V.7.0 (GraphPad Software, San Diego, California, USA).

\section{Patient and public involvement}

A patient and public involvement panel was not specifically recruited to inform the design, recruitment, conduct or dissemination plan for this study.

\section{Monitoring}

The researcher responsible for the interventions and control of the groups will monitor the research. During each session of intervention, participants will be assessed for vital signs and asked about any changes during activities that could result in the termination of the research.

The analyses will be based on the ITT principle, and they will include data on all randomised participants with at least one outcome measure. The significance level will be set at $95 \%(\mathrm{p}<0.05)$. All participants will be included in the analysis of the original groups following the CONSORT recommendations.

\section{OUTCOMES}

\section{Primary outcome}

\section{ALSFRS-R}

The ALSFRS-R will be administered through interviews with the volunteers at the end of the assessments. It consists of 12 items on a scale of $0-4$ with a total score ranging between 0 and 48 points, representing maximum disability and normal functioning, respectively. ${ }^{13}$ The ALSFRS-R will be quantified as a total score and with domain-specific subscores to compare the different moments of assessment and analysis of disease progression. ${ }^{28}$

\section{Number of exacerbations}

The exacerbation occurs with the impaired ability to cough and eliminate secretions from the airway during upper respiratory tract infections due to respiratory failure and concomitant weakness of the oropharyngeal muscles. The resulting bronchial mucus plug can decrease vital capacity and SpO2. Exacerbations are 
hypoventilation, and hypercapnia ${ }^{29}$ with $\mathrm{SpO}_{2}$ below $90 \%$, requiring emergency care. ${ }^{24}$

The episodes of emergency care that patients will encounter due to the worsening of their respiratory conditions will be counted to determine the efficiency of the service.

\section{PCF}

PCF and chest wall operating volumes will be assessed non-invasively through kinematics of the chest wall, using optoelectronic plethysmography (BTS Bioengineering, Italy). The system allows a three-dimensional assessment of the chest wall kinematics of a patient placed $90^{\circ}$ in the supine position using 52 retroreflective markers and 6 cameras ( 3 on the left and 3 on the right of the participant) that have been previously calibrated, following the described protocol. ${ }^{30}$ SmartCapture software will be used for data collection and for the three-dimensional model analysis and volumetric calculation for the quantitative analysis of operating volumes and PCF; DiaMov software (customised software designed and developed at the Politecnico di Milano, Italy) will be used.

\section{Secondary outcome}

Pulmonary function

The absolute and predicted FVC, forced expiratory volume in the 1st second $\left(\mathrm{FEV}_{1}\right)$ and $\mathrm{FEV}_{1} / \mathrm{FVC}$ will be measured with the patient seated using a Koko DigiDoser spirometer (nSpire Health, Longmont, USA) calibrated with a $3 \mathrm{~L}$ syringe. For execution, the American Thoracic Society (ATS) and European Respiratory Society (ERS) recommendations will be adopted. ${ }^{31}$ Analysis of the obtained values, based on the values proposed for assessing the normality of spirometric tests in healthy Brazilian adults, will be performed. ${ }^{32}$

\section{SNIP}

SNIP will be measured using a rigid material nasal plug, and one nostril will be occluded during the manoeuvre through the contralateral nostril. The plug will be connected to the NEPEB-LabCare/UFMG digital manovacuometer (Minas Gerais, Brazil) through a polyethylene catheter (internal diameter of approximately $1 \mathrm{~mm}$ and length of $72 \mathrm{~cm}$ ), and it will be comfortably and safely positioned in one of the nostrils to prevent it from falling during manoeuvres. The volunteer will be instructed to perform 12 manoeuvres of maximum inspiration through the contralateral nostril from the functional residual capacity while sitting and keeping the mouth closed. ${ }^{33}$ Verbal encouragement for strong and quick inspiration without hesitation will be provided at the end of observed exhalations preceded by breaths at the level of tidal volume. The following criteria will be used to choose the correctly developed manoeuvres: manoeuvres performed quickly and strongly, a total duration of SNIP of less than $500 \mathrm{~ms}$, pressure peak sustained for less than $50 \mathrm{~ms}$ and a smooth, descending respiratory curve without a biphasic peak. SNIP will be performed in the sitting position. The highest value obtained for the manoeuvres that meet the acceptance and reproducibility criteria will be used as a reference and for data analysis. The obtained values will be analysed based on the values proposed for healthy Brazilian adults. ${ }^{34}$

\section{SNEP}

The evaluator will measure nasal expiratory pressure through an oronasal mask worn by the patient to prevent air leakage from the sides; the mask will also be connected to the digital manometer vacuum NEPEB-LabCare/UFMG (Minas Gerais, Brazil) through a polyethylene catheter of dimensions previously described. The volunteer will be instructed to perform 10-20 maximum expiration manoeuvres, starting from the functional residual capacity. Verbal encouragement will be given at the end of inspiration, preceded by breaths at the tidal volume level, so that air will be released quickly and forcefully through the nose while the mouth is closed. The nasal expiratory pressure will be measured with the patient in a sitting position. The verbal command will be, 'loose strong', and the eligibility criteria for the manoeuvres will be the same as those used for the SNIP manoeuvres. The highest value that will meet the criteria will be used for data analysis and as a reference. The nasal expiratory pressure assessment technique was developed in our laboratory, based on previous clinical studies, and its methodological characteristics will be published soon. ${ }^{35}$

\section{MIP and MEP}

The strength of the respiratory muscles will be measured using a digital manometer (NEPEB-LabCare/UFMG, Brazil), following the recommendations of ATS/ERS (2002), with the volunteer in a sitting position. A disposable cylindrical mouthpiece with a $2 \mathrm{~mm}$ orifice will be used to minimise the pressure generated by the orofacial muscles during MEP and prevent glottis closure at the MIP. A nose clip will be used for both tests. Prediction equations will be used to analyse the data obtained. ${ }^{36}$ For MIP, the volunteer will start from the residual volume; the volunteer will be instructed to breathe at the functional residual capacity level and release as much air from the lungs as possible. Following this, a positive signal will be provided, and the evaluator will request maximum inspiration while simultaneously introducing the mouthpiece. For MEP, the manoeuvre will start from the total lung capacity. With the inverse command, the participant will be instructed to fill the lungs with air, and at the positive signal, the evaluator will attach the mouthpiece and request maximum expiration against airway occlusion. To prevent air leakage and the use of the orofacial muscles, the evaluator will press the volunteer's cheeks.

For each test, a minimum of three manoeuvres and a maximum of five will be performed, of which three will 
Table 1 Study schedule

\begin{tabular}{|c|c|c|c|c|c|c|c|}
\hline \multirow[b]{3}{*}{ Time point (months) } & \multicolumn{7}{|c|}{ Study period } \\
\hline & \multirow{2}{*}{$\begin{array}{l}\text { Enrolment } \\
-t_{1}\end{array}$} & \multirow{2}{*}{$\begin{array}{l}\text { Allocation } \\
0\end{array}$} & \multicolumn{2}{|c|}{ Post-allocation } & \multirow[b]{2}{*}{$6-9$} & \multirow[b]{2}{*}{$9-12$} & \multirow{2}{*}{$\begin{array}{l}\text { Close-out } \\
t_{x}\end{array}$} \\
\hline & & & $1-3$ & $3-6$ & & & \\
\hline \multicolumn{8}{|l|}{ Enrolment } \\
\hline Eligibility screen & $x$ & & & & & & \\
\hline Informed consent & $x$ & & & & & & \\
\hline (Phone contact) & & & $x$ & $x$ & $x$ & $x$ & \\
\hline Allocation/randomisation & & $x$ & & & & & \\
\hline \multicolumn{8}{|l|}{ Interventions } \\
\hline (Conventional respiratory care) & & & $x$ & $x$ & & & \\
\hline (Optimised respiratory care) & & & $x$ & $x$ & & & \\
\hline \multicolumn{8}{|l|}{ Assessments } \\
\hline (Peak cough flow) & $x$ & & $x$ & $x$ & & & $x$ \\
\hline (Forced vital capacity) & $x$ & & $x$ & $x$ & & & $x$ \\
\hline (Maximal respiratory pressure) & $x$ & & $x$ & $x$ & & & $x$ \\
\hline (Nasal respiratory pressures) & $x$ & & $x$ & $x$ & & & $x$ \\
\hline $\begin{array}{l}\text { (Amyotrophic Lateral Sclerosis Functional Rating } \\
\text { Scale-Revised) }\end{array}$ & $x$ & & $x$ & $x$ & $x$ & $x$ & $x$ \\
\hline
\end{tabular}

have to be acceptable (lasting at least $1.5 \mathrm{~s}$ ) and at least two reproducible (the values of the two major manoeuvres cannot differ by more than $10 \%$, and the value of the third-largest cannot differ by more than $20 \%$ from the largest value). Considering the learning effect, the last manoeuvre cannot have the highest value; if it happens, the exam will continue until a lower value is obtained. A minute's rest will be allowed between tests. The highest value obtained will be used for data analysis and as a reference.

The study schedule is shown in table 1 .

\section{ETHICS AND DISSEMINATION}

\section{Ethics approval and consent to participate}

The project was approved by the Research Ethics Committee of the University Hospital Onofre Lopes of Federal University of Rio Grande do Norte, Natal, Rio Grande do Norte, Brazil (CAAE: 11731019.5.0000.5292) and registered in the Brazilian Registry of Clinical Research-ReBEC with the number RBR-3z23ts on 7 August 2019. Updated: 12 March 2021. Patients and their caregivers will be invited to participate voluntarily and all information about randomised group and intervention will be given. After that, they will be required to sign IC term (online supplemental file).

\section{Consent for publication and confidentiality}

All information collected from the participants of the study will be kept confidential and stored in the laboratory's database, during and after the trial. They will only be accessed by the researchers to ensure anonymity and respect for human dignity and fulfil all the bioethics requirements of Resolution 466/2012 of the National Health Council and the Helsinki Declaration for research with humans.

Results of this study will be reported in full through peer-reviewed journals and presented at scientific conferences. Significant protocol modifications will be communicated to the participants, trial register and journals. A model of the informed consent form will be provided, if requested.

\section{Availability of data and materials}

The study protocol and data sets generated and/or analysed of all identified included participants during the current study will be available from the corresponding author on reasonable request to achieve the approved proposal aims.

\section{DISCUSSION}

Individuals with ALS have respiratory complications that worsen rapidly, and this can culminate in death. These patients should be provided with regular daily respiratory care and assistance by caregivers and family members as well as professional monitoring to reduce exacerbations.

The challenges associated with public health assistance for patients as well as the lack of knowledge among health professionals and the community favours the worsening of ALS and consequently increased hospitalisations and public spending.

We hope that the study can demonstrate the importance of home-based physiotherapy with specialised assistance, 
which will provide patients and their families the convenience and safety. We believe that a well-implemented therapeutic programme will reduce the morbidity and mortality rates of patients with ALS.

\section{Author affiliations}

${ }^{1}$ Departamento de Fisioterapia, PneumoCardioVascular Lab/HUOL e Laboratório de Inovação Tecnológica em Reabilitação, Hospital Universitário Onofre Lopes, Empresa Brasileira de Serviços Hospitalares (EBSERH), Universidade Federal do Rio Grande do Norte, Natal, Brazil

${ }^{2}$ Medicina Integrada, Hospital Universitário Onofre Lopes, Empresa Brasileira de Serviços Hospitalares (EBSERH), Universidade Federal do Rio Grande do Norte, Natal, Brazil

${ }^{3}$ Departamento de Kinesiología, Universidad de Chile, Santiago de Chile, Chile

\section{Twitter Karen Pondofe @karenpondofe and Vanessa R Resqueti @vanessaresqueti}

Contributors KP, research concept and study design, literature review, data collection, data analysis and interpretation, statistical analyses, writing of the manuscript and reviewing. GAFF, data analysis and interpretation, statistical analyses and reviewing. OB, data collection. MEDJ, research concept and study design, data interpretation, review of the manuscript. RT-C, data analysis and interpretation, reviewing. VRR, research concept and study design, data analysis and interpretation, statistical analyses, writing of the manuscript and reviewing.

Funding This work is supported by the Ministry of Health through TED 132/2018, GESCOM grant number 2500.194604/2018-76 and the support of the Coordenação de Aperfeiçoamento de Pessoal de Nível Superior—Brazil (CAPES)—Funding Code 001. VRR is a fellow of the Conselho Nacional de Desenvolvimento Científico e Tecnológico (CNPq) - process number 315580/2018-6. GAFF is a fellow of the Conselho Nacional de Desenvolvimento Científico e Tecnológico (CNPq) — process number 312876/2018-1.

\section{Competing interests None declared.}

Patient consent for publication Not required.

Provenance and peer review Not commissioned; externally peer reviewed.

Supplemental material This content has been supplied by the author(s). It has not been vetted by BMJ Publishing Group Limited (BMJ) and may not have been peer-reviewed. Any opinions or recommendations discussed are solely those of the author(s) and are not endorsed by BMJ. BMJ disclaims all liability and responsibility arising from any reliance placed on the content. Where the content includes any translated material, BMJ does not warrant the accuracy and reliability of the translations (including but not limited to local regulations, clinical guidelines, terminology, drug names and drug dosages), and is not responsible for any error and/or omissions arising from translation and adaptation or otherwise.

Open access This is an open access article distributed in accordance with the Creative Commons Attribution Non Commercial (CC BY-NC 4.0) license, which permits others to distribute, remix, adapt, build upon this work non-commercially, and license their derivative works on different terms, provided the original work is properly cited, appropriate credit is given, any changes made indicated, and the use is non-commercial. See: http://creativecommons.org/licenses/by-nc/4.0/.

\section{ORCID iD}

Karen Pondofe http://orcid.org/0000-0003-2105-1096

\section{REFERENCES}

1 EFNS Task Force on Diagnosis and Management of Amyotrophic Lateral Sclerosis:, Andersen PM, Abrahams S, et al. EFNS guidelines on the clinical management of amyotrophic lateral sclerosis (MALS)-revised report of an EFNS task force. Eur J Neurol 2012;19:360-75.

2 Perrin C, Unterborn JN, Ambrosio Carolyn D', et al. Pulmonary complications of chronic neuromuscular diseases and their management. Muscle Nerve 2004;29:5-27.

3 Lui AJ, Byl NN. A systematic review of the effect of moderate intensity exercise on function and disease progression in amyotrophic lateral sclerosis. J Neurol Phys Ther 2009;33:68-87.

4 Chiò A, Logroscino G, Traynor BJ, et al. Global epidemiology of amyotrophic lateral sclerosis: a systematic review of the published literature. Neuroepidemiology 2013;41:118-30.
5 Prado LdeGR, Bicalho ICS, Vidigal-Lopes M, et al. Amyotrophic lateral sclerosis in Brazil: case series and review of the Brazilian literature. Amyotroph Lateral Scler Frontotemporal Degener 2016;17:282-8.

6 Dietrich-Neto F, Callegaro D, Dias-Tosta E, et al. Amyotrophic lateral sclerosis in Brazil: 1998 national survey. Arq Neuropsiquiatr 2000:58:607-15.

7 Moraes L, Goldbaum M, Silva HCA, et al. Incidence rate of amyotrophic lateral sclerosis (ALS) in Sao Paulo City, Brazil, 1991-1997. Arq Neuro-Psiquiatr 1998;56:343.

8 MND Guideline Development Group. Guidelines for the physiotherapy management of motor neuron disease (MND. Dublin: Irish Hospice Foundation, 2014.

9 Swinnen B, Robberecht W. The phenotypic variability of amyotrophic lateral sclerosis. Nat Rev Neurol 2014;10:661-70.

10 Magnus T, Beck M, Giess R, et al. Disease progression in amyotrophic lateral sclerosis: predictors of survival. Muscle Nerve 2002;25:709-14.

11 Andrews JA, Meng L, Kulke SF, et al. Association between decline in slow vital capacity and respiratory insufficiency, use of assisted ventilation, tracheostomy, or death in patients with amyotrophic lateral sclerosis. JAMA Neurol 2018;75:58-64.

12 Polkey MI, Lyall RA, Yang K, et al. Respiratory muscle strength as a predictive biomarker for survival in amyotrophic lateral sclerosis. Am J Respir Crit Care Med 2017;195:86-95.

13 Cedarbaum JM, Stambler N, Malta E, et al. The ALSFRS-R: a revised ALS functional rating scale that incorporates assessments of respiratory function. BDNF ALS Study Group (phase III). J Neurol Sci 1999;169:13-21.

14 Hardiman Oet al. Esclerose lateral amiotrófica. Nat Rev Dis Primers 2017;3:17071.

15 Laghi F, Tobin MJ. Disorders of the respiratory muscles. Am J Respir Crit Care Med 2003;168:10-48.

16 Kravitz RM. Airway clearance in Duchenne muscular dystrophy. Pediatrics 2009;123 Suppl 4:S231-5.

17 Smith JA, Aliverti A, Quaranta M, et al. Chest wall dynamics during voluntary and induced cough in healthy volunteers. $J$ Physiol 2012;590:563-74.

18 Lo Mauro A, Aliverti A. Physiology of respiratory disturbances in muscular dystrophies. Breathe 2016;12:318-27.

19 Macpherson CE, Bassile CC. Pulmonary physical therapy techniques to enhance survival in amyotrophic lateral sclerosis: a systematic review. J Neurol Phys Ther 2016;40:165-75.

20 Chan A-W, Tetzlaff JM, Altman DG, et al. Spirit 2013 statement: defining standard protocol items for clinical trials. Ann Intern Med 2013;158:200-7.

21 Brooks BR, Miller RG, Swash M, et al. El Escorial revisited: revised criteria for the diagnosis of amyotrophic lateral sclerosis. Amyotrophic Lateral Sclerosis and Other Motor Neuron Disorders 2000;1:293-9.

22 Branco LMT, Zanao T, De Rezende TJ, et al. Transcultural validation of the ALS-CBS cognitive section for the Brazilian population. Amyotroph Lateral Scler Frontotemporal Degener 2017;18:60-7.

23 World Health Organization. Global health estimates 2016: deaths by cause, age, sex, by country and by region, 2000-2016. Geneva, 2018.

24 Malachias MVB, Souza W, Plavnik FL. Vii Brazilian guidelines on dyslipidemias and prevention of atherosclerosis. Arq Bras Cardiol 2016;107:1-22.

25 Kyung WJ, Sook JL, Sang BK. Effects of mechanical inspiration and expiration exercise on velopharyngeal incompetence in subacute stroke patients. J Rehabil Med 2018.

26 Faul F, Erdfelder E, Buchner A, et al. Statistical power analyses using G*Power 3.1: tests for correlation and regression analyses. Behav Res Methods 2009;41:1149-60.

27 Kimura F, Fujimura C, Ishida S, et al. Progression rate of ALSFRS-R at time of diagnosis predicts survival time in ALS. Neurology 2006;66:265-7.

28 Rooney J, Burke T, Vajda A, et al. What does the ALSFRS-R really measure? A longitudinal and survival analysis of functional dimension subscores in amyotrophic lateral sclerosis. J Neurol Neurosurg Psychiatry 2017;88:381-5.

29 Francis K, Bach JR, DeLisa JA. Evaluation and rehabilitation of patients with adult motor neuron disease. Arch Phys Med Rehabil 1999;80:951-63.

30 Aliverti $A$, Dellacà R, Pelosi $P$, et al. Compartmental analysis of breathing in the supine and prone positions by optoelectronic plethysmography. Ann Biomed Eng 2001;29:60-70.

31 Miller MR, Hankinson J, Brusasco V. ATS/ERS. Task Force. Standardisation of spirometry. Eur Respir J 2005;26:319-38. 
32 Pereira C, Sato T, Rodrigues SC. New reference values for forced spirometry in adult white Brazilians. J Bras de Pneumol 2007;33:397-406.

33 Lofaso F, Nicot F, Lejaille M, et al. Sniff nasal inspiratory pressure: what is the optimal number of sniffs? Eur Respir $J$ 2006;27:980-2.

34 Araújo PRS, Resqueti VR, Nascimento Junior J, et al. Reference values for sniff nasal inspiratory pressure in healthy subjects in Brazil: a multicenter study. J Bras Pneumol 2012;38:700-7.
35 Ichikawa T, Yokoba M, Kimura M, et al. Genioglossus muscle activity during sniff and reverse sniff in healthy men. Exp Physiol 2018;103:1656-65.

36 Neder JA, Andreoni S, Lerario MC, et al. Reference values for lung function tests. II. maximal respiratory pressures and voluntary ventilation. Braz J Med Biol Res 1999;32:719-27. 